\title{
Stability and Feigenbaum's Universality in Two Dimensional Chaotic Map
}

\author{
${ }^{1}$ Tarini Kumar Dutta, ${ }^{2}$ Debasish Bhattacharjee \\ ${ }^{1}$ Gauhati University; Guwahati 781014; INDIA \\ ${ }^{2}$ B.Borooah College Guwahati 781016; INDIA
}

\begin{abstract}
In this paper a two dimensional non linear map is taken whose period doubling dynamical behavior has been analyzed. The bifurcation points have been calculated numerically and have been observed that the map follows a universal behavior that has been proposed by Feigenbaum. With the help of experimental bifurcation points the accumulation point where chaos starts has been calculated.
\end{abstract}

Key Words: Period-Doubling Bifurcation/ Periodic orbits / Feigenbaum Universal Constant / Accumulation point

\section{Introduction}

A very simple discrete model of the interaction between predator $\mathrm{P}$ and prey $\mathrm{N}$, where it is assumed that predator can consume prey without limit, is given by

$$
N_{t+1}=r N_{t} e^{-b P_{t}} \quad P_{t+1}=N_{t}\left(1-e^{-a P_{t}}\right),
$$

where $\mathrm{a}, \mathrm{b}, \mathrm{r}>0$. Murray[10,11] gave a special case of the above model taking $\mathrm{a}=\mathrm{b}$ and it is noticed that the model is unrealistic in the sense that the solutions can grow unboundedly with t. Hone, Irle and Thurura[7] discussed some of the beautiful aspects of dynamical behavior of the above said model and they have suggested a more realistic model

$x_{n+1}=x_{n} e^{r\left(1-\frac{x_{n}}{k}\right)-b y_{n}}$

$y_{n+1}=x_{n}\left(1-e^{-a y_{n}}\right)$

where $\mathrm{a}, \mathrm{b}, \mathrm{c}, \mathrm{k}, \mathrm{r}$ are adjustable parameters.

It is realistic in the sense that it represents Ricker curve $x_{n+1}=x_{n} e^{r\left(1-\frac{x_{n}}{k}\right)}$ when restricted to one dimension. In case $\mathrm{a}=\mathrm{b}$ it was discussed by Beddington et.al[1] which may be called as density dependent model. In this paper, we want to explore some dynamical aspects of the two dimensional ecological map (1.1.1).

In section 1.2 a particular case of the map is taken and detailed dynamical behavior has been studied with the help of bifurcation diagram and Feigenbaum's theory. It is observed that the map undergoes period doubling bifurcation and ultimately takes chaotic structure. In section 1.3 certain numerical techniques have been adopted to formulate some computer programming which has become useful in calculating bifurcation points. Further with the help of the numerical data it has been verified that the map obeys Feigenbaum universal constant $\delta$. Lastly in section 1.4 using $\delta$, the accumulation point is calculated.

\subsection{Dynamical behaviour of a particular map from its class:}

We now fix some of the parameters say $a, b, k$ and keep varying $r$ to analyse the detailed dynamical behaviour of the map. Let us take $a=0.5105854, b=0.01, k=4.7913155$. On inspection it can be seen that $(0,0)$ and $(k, 0)$ are two fixed points of the model satisfying the equation

$$
\begin{aligned}
& f(x, y)=(x, y)=\left(x e^{r\left(1-\frac{x}{k}\right)-b y}, x\left(1-e^{-a y}\right)\right) \\
& \text { i.e. } \quad \begin{array}{rl}
x & x\left(1-\frac{x}{k}\right)-b y \\
y & =x\left(1-e^{-a y}\right)
\end{array}
\end{aligned}
$$

Using "Mathematica" we generate the bifurcation diagram for the observation of the whole dynamical behaviour of the map as $r$ is varied. 


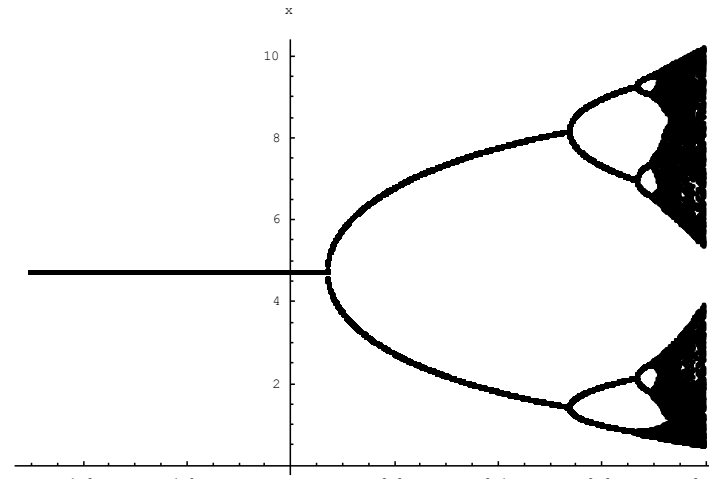

Fig 1.2.a: The figure is generated using 100 points which are taken after iterating 5000 points of the map at every parameter value of $r$, and plotted the $\mathrm{x}$ coordinate of the point $(\mathrm{x}, \mathrm{y})$ vs. $\mathrm{r}$.

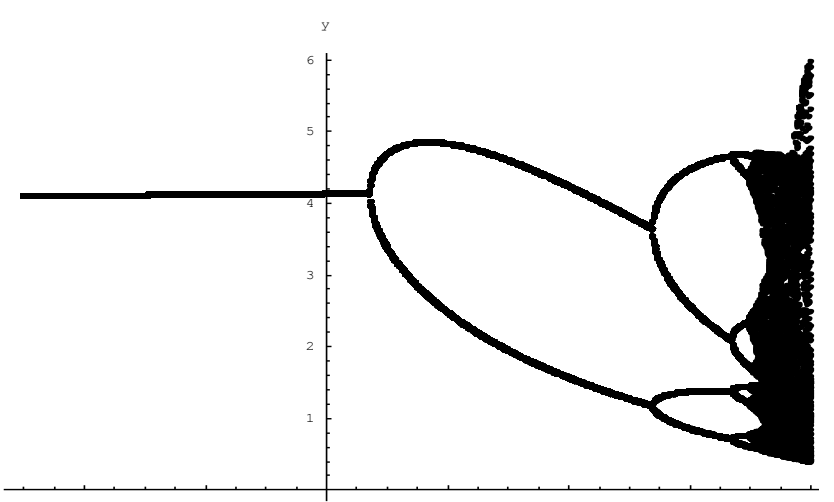

Fig 1.2.b: The figure is generated using 100 points which is taken after iterating 5000 points of the map at every parameter value of $r$, and plotted the $y$ coordinate of the point $(x, y)$ vs. $r$.

From the diagram it has been clear that the map follows period doubling route to chaos as " $\mathrm{r}$ " is varied. We know that $(0,0)$ is unstable for $(1.1 .1)$.Also $(k, 0)$ is unstable as ak>1 in this case. Let $\left(\mathrm{x}_{0}, \mathrm{y}_{0}\right)$ be a fixed point of the map $\mathrm{f}$ where neither of $\mathrm{x}_{0}, \mathrm{y}_{0}$ are equal to zero.

The fixed point $\left(\mathrm{x}_{0}, \mathrm{y}_{0}\right)$ remains stable for all values of $\mathrm{r}$ lying in the interval say $\mathrm{I}_{1}$ and a stable periodic trajectory of period one appears around it till the two eigenvalues of the Jacobian matrix $\mathrm{J}$ at $\left(\mathrm{x}_{0}, \mathrm{y}_{0}\right)$ remains less than one in modulus, and as a result all the neighbouring points (that is, points in the domain of attraction) are attracted towards $\left(\mathrm{x}_{0}, \mathrm{y}_{0}\right), \mathrm{r}$ lying in $\mathrm{I}_{1}$. However the least upper bound of the interval $\mathrm{I}_{1}$ can be obtained from the equations (1.2.1) and $\min \left\{\lambda_{1}, \lambda_{2}\right\}=-1$. If we now begin to increase the value of $\mathrm{r}$ exceeding the l.u.b. of $\mathrm{I}_{1}$, then it happens that one of the eigenvalues starts decreasing through -1 and the fixed point $\left(\mathrm{x}_{0}, \mathrm{y}_{0}\right)$ loses its stability .Hence $r_{1}=1 . u . b$. of $I_{1}$ is the point where the first bifurcation occurs. So, if we keep on increasing the value of $r$ the point $\left(\mathrm{x}_{0}, \mathrm{y}_{0}\right)$ becomes unstable and there arises around it two points, say, $\left(\mathrm{x}_{21}(\mathrm{r}), \mathrm{y}_{21}(\mathrm{r})\right)$ and $\left(\mathrm{x}_{22}(\mathrm{r})\right.$, $\mathrm{y}_{22}(\mathrm{r})$ ) forming a stable periodic trajectory of period 2. All the neighbouring points except the stable manifold of $\left(\mathrm{x}_{0}(\mathrm{r}), \mathrm{y}_{0}(\mathrm{r})\right)$ are attracted towards these two points. Since the period emerged becomes double, the previous eigenvalue which was -1 becomes +1 and as we keep increasing $r$, one of the eigenvalues starts decreasing from +1 to -1 . When we reach a certain value of $r$, we find that one of the eigenvalues of the Jacobian of $f^{2}$ (because of the chain rule of differentiation, it does not matter at which periodic point one evaluates the eigenvalues) becomes -1 , indicating the loss of stability of the periodic trajectory of period two. Thus, the second bifurcation takes place at this value $r_{2}$ of $r$. We can then repeat the same arguments, and find that the periodic trajectory of period 2 becomes unstable and a periodic trajectory of period 4 appears in its neighbourhood. This phenomenon continues up to a particular value of $r$ say $r_{3}$, at which the periodic trajectory of period 4 losses its stability in such a way that one of the eigenvalues at any of its periodic points become -1 , and thus it gives the third bifurcation at $\mathrm{r}_{3}$.

Increasing the value further and further, and repeating the same arguments we obtain a sequence $\left\{\mathrm{r}_{\mathrm{n}}\right\}$ as bifurcation values for the parameter $r$ such that at $r=r_{n}$ a periodic trajectory of period $2^{\mathrm{n}}$ arises and all periodic trajectories of period $2^{\mathrm{m}}(\mathrm{m}<\mathrm{n})$ remain unstable. The sequence $\left\{\mathrm{r}_{\mathrm{n}}\right\}$ behaves in a universal manner such that $\mathrm{r}_{\alpha^{-}} \mathrm{r}_{\mathrm{n}} \sim \mathrm{c} \delta^{-\mathrm{n}}$, where $\mathrm{c}$ is independent of $\mathrm{n}$ and $\delta$ and is the Feigenbaum Universal constant. For the dissipative case, that is, contraction of area $\delta$ equals $4.6692016091029 \ldots$ and for the conservative case, i.e. the preservation of area $\delta$ equals $8.721097200 \ldots$. 
Furthermore, the Feigenbaum theory says that the our map $\mathrm{f}$ at $\mathrm{r}=\mathrm{r}{ }_{\infty}$ has an invariant set $\mathrm{F}$ of Cantor type encompassed by infinitely many unstable periodic orbits of period $2^{n}(n=0,1,2, \ldots)$, and that all the neighbouring points except those belonging to these unstable orbits and their stable manifolds are attracted to cantor like set $\mathrm{F}$ under the iterations of $\mathrm{f}$ at the parameter $\mathrm{r}$.

\subsection{Numerical Method for Obtaining Periodic Points:}

Although there are so many sophisticated numerical algorithms to find a periodic fixed point, we have found that the Newton Recurrence formula is one of the best numerical methods with negligible error for our purpose. Moreover, it gives fast convergence of a periodic fixed point.

The Newton Recurrence formula is

$$
\overline{x_{n+1}}=\overline{x_{n}}-D f\left(\overline{x_{n}}\right)^{-1} f\left(\overline{x_{n}}\right) \text {, where } \mathrm{n}=0,1,2, \ldots \text { and } D f(\bar{x}) \text { is the Jacobian of the map } \mathrm{f} \text { at }
$$

the vector $\bar{x}$. We see that this map $\mathrm{f}$ is equal to $\mathrm{f}^{\mathrm{k}}-\mathrm{I}$ in our case, where $\mathrm{k}$ is the appropriate period. The Newton formula actually gives the zero(s) of a map, and to apply this numerical tool in our map one needs a number of recurrence formulae which are given below.

Let the initial point be $\left(\mathrm{x}_{0}, \mathrm{y}_{0}\right)$,

Then,

$$
\begin{aligned}
\mathrm{f}\left(\mathrm{x}_{0}, \mathrm{y}_{0}\right)=\left(x_{0} e^{r\left(1-\frac{x_{0}}{k}\right)-b y_{0}}, x_{0}\left(1-e^{-a y_{0}}\right)\right)=\left(\mathrm{x}_{1}, \mathrm{y}_{1}\right) \\
\\
\mathrm{f}^{2}\left(\mathrm{x}_{0}, \mathrm{y}_{0}\right)=\mathrm{f}\left(\mathrm{x}_{1}, \mathrm{y}_{1}\right)=\left(\mathrm{x}_{2}, \mathrm{y}_{2}\right) \\
\text { where } \mathrm{x}_{2}=x_{1} e^{r\left(1-\frac{x_{1}}{k}\right)-b y_{1}}, \mathrm{y}_{2}=x_{1}\left(1-e^{-a y_{1}}\right)
\end{aligned}
$$

Proceeding in this manner the following recurrence formula for our map can be established.

$$
\mathrm{x}_{\mathrm{n}}=x_{n-1} e^{r\left(1-\frac{x_{n}-1}{k}\right)-b y_{n-1}} \text { and } \mathrm{y}_{\mathrm{n}}=x_{n-1}\left(1-e^{-a y_{n-1}}\right) \quad \text { where } \mathrm{n}=1,2,3 \ldots
$$

Since the Jacobian of $\mathrm{f}^{\mathrm{k}}$ ( $\mathrm{k}$ times iteration of the map ) is the product of the Jacobian of each iteration of the map, we proceed as follows to describe our recurrence mechanism for the Jacobian matrix.

The Jacobian $\mathrm{J}_{1}$ for the transformation

$$
\begin{aligned}
& \mathrm{f}\left(\mathrm{x}_{0}, \mathrm{y}_{0}\right)=\left(x_{0} e^{r\left(1-\frac{x_{0}}{k}\right)-b y_{0}}, x_{0}\left(1-e^{-a y_{0}}\right)\right) \text { is }
\end{aligned}
$$

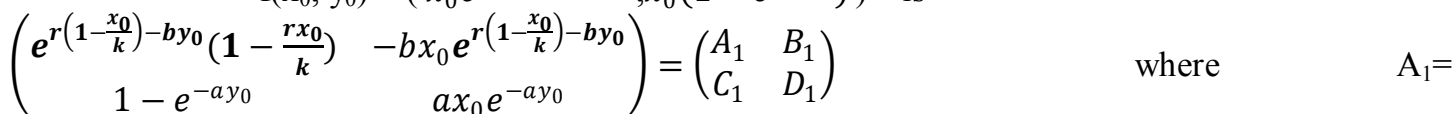

$$
\begin{aligned}
& \boldsymbol{e}^{\boldsymbol{r}\left(\mathbf{1}-\frac{x_{0}}{\boldsymbol{k}}\right)-\boldsymbol{b} y_{0}}\left(\mathbf{1}-\frac{\boldsymbol{r} x_{\mathbf{0}}}{\boldsymbol{k}}\right), \mathrm{B}_{1}=-b x_{0} \boldsymbol{e}^{\boldsymbol{r}\left(\mathbf{1}-\frac{x_{\mathbf{0}}}{\boldsymbol{k}}\right)-\boldsymbol{b} y_{0}}, \mathrm{C}_{1}=1-e^{-a y_{0}}, \quad \mathrm{D}_{1}=a x_{0} e^{-a y_{0}} .
\end{aligned}
$$

Next the Jacobian $\mathrm{J}_{2}$ for the transformation

$\mathrm{f}^{2}\left(\mathrm{x}_{0}, \mathrm{y}_{0}\right)=\left(\mathrm{x}_{2}, \mathrm{y}_{2}\right)$ where $\mathrm{x}_{2}$ and $\mathrm{y}_{2}$ are as mentioned above, is the product of the Jacobians for the transformations

$$
\begin{aligned}
& \mathrm{f}\left(\mathrm{x}_{1}, \mathrm{y}_{1}\right)=\left(x_{1} e^{r\left(1-\frac{x_{1}}{k}\right)-b y_{1}}, x_{1}\left(1-e^{-a y_{1}}\right)\right) \text { and } \\
& \mathrm{f}\left(\mathrm{x}_{0}, \mathrm{y}_{0}\right)=\left(x_{0} e^{r\left(1-\frac{x_{0}}{k}\right)-b y_{0}}, x_{0}\left(1-e^{-a y_{0}}\right)\right)=\left(\mathrm{x}_{1}, \mathrm{y}_{1}\right) \\
& \text { So we obtain }
\end{aligned}
$$

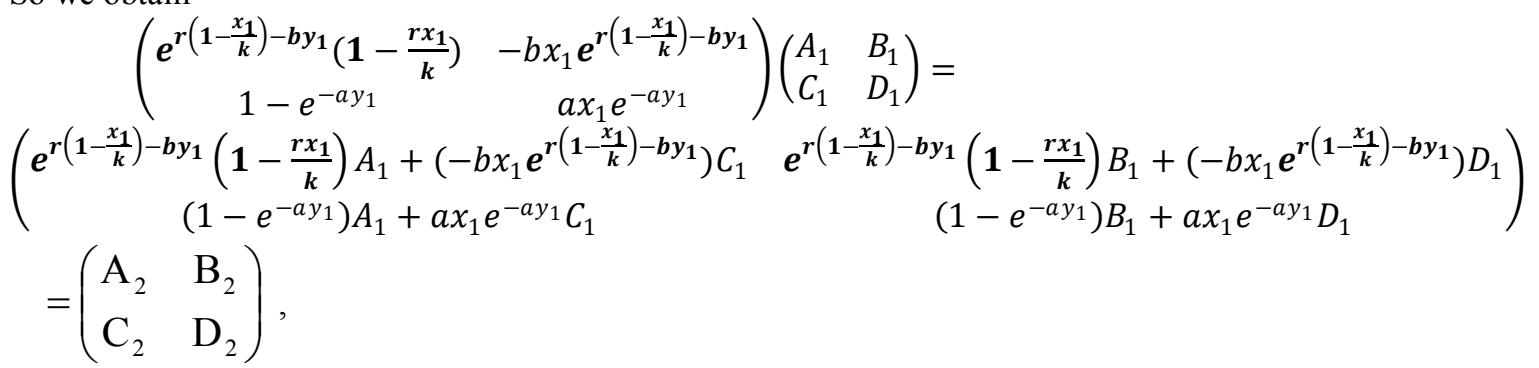

where $\mathrm{A}_{2}=e^{r\left(1-\frac{x_{1}}{k}\right)-b y_{1}}\left(1-\frac{r x_{1}}{k}\right) A_{1}+\left(-b x_{1} e^{r\left(1-\frac{x_{1}}{k}\right)-b y_{1}}\right) C_{1}$,

$\mathrm{B}_{2}=e^{r\left(1-\frac{x_{1}}{k}\right)-b y_{1}}\left(1-\frac{r x_{1}}{k}\right) B_{1}+\left(-b x_{1} e^{r\left(1-\frac{x_{1}}{k}\right)-b y_{1}}\right) D_{1}$

$\mathrm{C}_{2}=\left(1-\mathrm{e}^{-\mathrm{ay}_{1}}\right) \mathrm{A}_{1}+\mathrm{ax}_{1} \mathrm{e}^{-\mathrm{ay}_{1}} \mathrm{C}_{1}, \mathrm{D}_{2}=\left(1-e^{-a y_{1}}\right) B_{1}+a x_{1} e^{-a y_{1}} D_{1}$.

Continuing this process in this way we have the Jacobian for $\mathrm{f}^{\mathrm{m}}$ as

$$
\mathrm{J}_{\mathrm{m}}=\left(\begin{array}{ll}
\mathrm{A}_{\mathrm{m}} & \mathrm{B}_{\mathrm{m}} \\
\mathrm{C}_{\mathrm{m}} & \mathrm{D}_{\mathrm{m}}
\end{array}\right)
$$

with a set of recursive formula as 
$\mathrm{A}_{\mathrm{m}}=\boldsymbol{e}^{r\left(1-\frac{\boldsymbol{x}_{m-1}}{k}\right)-\boldsymbol{b} y_{m-1}}\left(1-\frac{r x_{m-1}}{k}\right) A_{m-1}+\left(-b x_{m-1} e^{r\left(1-\frac{x_{m-1}}{k}\right)-b y_{m-1}}\right) C_{m-1} \quad, \quad \mathrm{~B}_{\mathrm{m}}=\boldsymbol{e}^{r\left(1-\frac{x_{m-1}}{k}\right)-b y_{m-1}}(1-$ $\boldsymbol{r x m}-\mathbf{1} \boldsymbol{k A m}-1+\left(-b x m-1\right.$ er $\left.\mathbf{1}-\boldsymbol{x m}-\mathbf{1 k}-\boldsymbol{b y m}^{-1}\right) \mathrm{Cm}^{-1}, \mathrm{C}_{\mathrm{m}}=1-\mathrm{e}-\mathrm{aym}-1 \mathrm{Am}-1+\mathrm{axm}-1 \mathrm{e}-\mathrm{aym}-1 \mathrm{Cm}-1$, $\mathrm{D}_{\mathrm{m}}=\left(1-e^{-a y_{m-1}}\right) B_{m-1}+a x_{m-1} e^{-a y_{m-1}} D_{m-1} \quad(\mathrm{~m}=2,3,4,5 \ldots)$.

Since the fixed point of this map $\mathrm{f}$ is a zero of the map $F(x, y)=f(x, y)-(x, y)$, the Jacobian of $F^{(k)}$ is given by

$J_{k}-I=\left(\begin{array}{cc}A_{k}-1 & B_{k} \\ C_{k} & D_{k}-1\end{array}\right)$. Its inverse is $\left(J_{k}-I\right)^{-1}=\frac{1}{\Delta}\left(\begin{array}{cc}D_{k}-1 & -B_{k} \\ -C_{k} & A_{k}-1\end{array}\right)$

Where $\Delta=\left(A_{k}-1\right)\left(D_{k}-1\right)-B_{k} C_{k}$, the Jacobian determinant. Therefore, Newton's method gives the following recurrence formula in order to yield a periodic point of $F^{k}$

$$
\begin{gathered}
\mathrm{x}_{\mathrm{n}+1}=\mathrm{x}_{\mathrm{n}}-\frac{\left(\mathrm{D}_{\mathrm{k}}-1\right)\left(\overline{\mathrm{x}_{\mathrm{n}}}-\mathrm{x}_{\mathrm{n}}\right)-\mathrm{B}_{\mathrm{k}}\left(\overline{\mathrm{y}_{\mathrm{n}}}-\mathrm{y}_{\mathrm{n}}\right)}{\Delta} \\
\mathrm{y}_{\mathrm{n}+1}=\mathrm{y}_{\mathrm{n}}-\frac{\left(-\mathrm{C}_{\mathrm{k}}\right)\left(\overline{\mathrm{x}_{\mathrm{n}}}-\mathrm{x}_{\mathrm{n}}\right)+\left(\mathrm{A}_{\mathrm{k}}-1\right)\left(\overline{\mathrm{y}_{\mathrm{n}}}-\mathrm{y}_{\mathrm{n}}\right)}{\text { where } \mathrm{F}^{\mathrm{k}}\left(\overline{x_{n}}\right)=\left(x_{n}, y_{n}\right)}
\end{gathered}
$$

\subsection{Numerical Methods for Finding Bifurcation Values:}

As

described above for some particular value of $r=r_{1}$ say we calculate the fixed point of $f^{k}$ and hence calculate the eigenvalues of $\mathrm{J}_{\mathrm{k}}$ at the fixed point. Let $\left(\mathrm{x}_{1}, \mathrm{y}_{1}\right),\left(\mathrm{x}_{2}, \mathrm{y}_{2}\right), \ldots\left(\mathrm{x}_{\mathrm{k}}, \mathrm{y}_{\mathrm{k}}\right)$ be the periodic points of $\mathrm{f}$ at $\mathrm{r}_{1}$. Let $\lambda_{1}, \lambda_{2}$ be the two eigen values of $\mathrm{J}_{\mathrm{k}}$ at $\mathrm{r}_{1}$, let $\mathrm{I}\left(\mathrm{k}, \mathrm{r}_{1}\right)=\min \left\{\lambda_{1}, \lambda_{2}\right\}$, where $\mathrm{n}=2^{\mathrm{k}}$ is the period number . Then we search two values of " $r$ " say $r_{11}$ and $r_{22}$ such that $\left(I\left(k, r_{11}\right)+1\right)\left(I\left(k, r_{22}\right)+1\right)<0$. Then the existence of $k^{\text {th }}$ bifurcation point is confirmed in between $r_{11}$ and $r_{22}$. Then we may apply some of the numerical techniques viz. Bisection method or Regula Falsi method on $r_{11}$ and $r_{22}$ for sufficient number of iterations to get $r$ such that $\mathrm{I}(\mathrm{k}, \mathrm{r})=-1$.

\begin{tabular}{|c|c|c|}
\hline Bifurcation Point & One of the Periodic Points & Eigen Values \\
\hline 2.07316614342238 & $\{4.69599923494547,4.12426302606314\}$ & $\begin{array}{l}(0.25999664774492,- \\
1.00000000000000)\end{array}$ \\
\hline 2.54050129070967 & $\{8.11608700086330,1.16038287512164\}$ & $\begin{array}{l}(0.31180912242995,- \\
1.00000000000000)\end{array}$ \\
\hline 2.67116687196673 & $\{6.91544986038299,0.69773150582828\}$ & $\begin{array}{l}0.09490165865225,- \\
1.00000000000002\end{array}$ \\
\hline 2.69891673464453 & $\{7.34865429449885,0.74734847008588\}$ & $\begin{array}{l}0.01559292499667,- \\
1.00000000000000\end{array}$ \\
\hline 2.70492837744115 & $\{7.51308973489738,0.77205507079669\}$ & $\begin{array}{l}0.00006321861390,- \\
0.99999999999985\end{array}$ \\
\hline 2.70621613564954 & $\{7.54912965735809,0.77702394568493\}$ & $\begin{array}{l}0.00000006162761,- \\
1.00000000000271\end{array}$ \\
\hline 2.70649204147512 & $\{7.21450548296841,0.71317442047950\}$ & $\begin{array}{l}-0.0000000,- \\
1.00000000000038\end{array}$ \\
\hline 2.70655113344213 & $\{7.26474745250725,0.72219289121455\}$ & $\begin{array}{l}0.00000000000000,- \\
0.99999999996327\end{array}$ \\
\hline 2.70656378927931 & $\{7.27520342957596,0.72408436411426\}$ & $\begin{array}{l}0.00000000000000,- \\
1.00000000013870\end{array}$ \\
\hline 2.70656649977671 & $\{7.27349102349901,0.72376847377666\}$ & $\begin{array}{l}-0.00000000000000,- \\
0.99999999599786\end{array}$ \\
\hline 2.70656708028252 & $\{7.27548585283783,0.72413197700605\}$ & $\begin{array}{l}-0.00000000000000,- \\
0.99999996614424\end{array}$ \\
\hline 2.70656720460910 & $\{7.27546245940362,0.72412755512584\}$ & $\begin{array}{l}0.00000000000000,- \\
1.00000003749797\end{array}$ \\
\hline 2.70656723123604 & $\{7.27546688390153,0.72412833107571\}$ & $\begin{array}{l}-0.00000000000000,- \\
0.99999912937029\end{array}$ \\
\hline 2.70656723693871 & $\{7.27530687414260,0.72409910357573\}$ & $\begin{array}{l}0.00000000000000,- \\
0.99999255147187\end{array}$ \\
\hline 2.70656723816000 & $\{7.27546709616816,0.72412836150319\}$ & $\begin{array}{l}0.00000000000000,- \\
0.99989593404106\end{array}$ \\
\hline
\end{tabular}

Our numerical results are as follows:

Table 1.4.a: Bifurcation points and one of the periodic points. 
The Feigenbaum universal constant is calculated using the experimentally calculated bifurcation point using the following formula $\delta_{\mathrm{n}}=\frac{A_{\mathrm{n}}-A_{\mathrm{n}-1}}{\mathrm{~A}_{\mathrm{n}+1}-\mathrm{A}_{\mathrm{n}}}$, where $A_{n}$ represents $\mathrm{n}^{\text {th }}$ bifurcation point. The values of $\delta_{n}$ are as follows.

$\delta_{1}=3.5765742040966133$

$\delta_{2}=4.7086928960405725$

$\delta_{3}=4.616019882851797$

$\delta_{4}=4.668300894883369$

$\delta_{5}=4.6673831756985305$

$\delta_{6}=4.669091917930064$

$\delta_{7}=4.669147221892675$

$\delta_{8}=4.669193625004722$

$\delta_{9}=4.669199433935363$

$\delta_{10}=4.669201142869103$

$\delta_{11}=4.669202741401066$

It may be observed that the map obeys Feigenbaum universal behaviour as the sequence $\left\{\delta_{n}\right\}$ converges to $\delta$ as $\mathrm{n}$ becomes very large.

\subsection{Accumulation Point:}

As discussed in chapter 2 , the accumulation point can be calculated by the formula $A_{\infty}=\left(A_{2}-A_{1}\right) /(\delta$ 1 ), where $\delta$ is Feigenbaum constant. But it has been observed that $\left\{\delta_{n}\right\}$ converges to $\delta$ as $n \rightarrow \infty$. Therefore a sequence of accumulation point $\left\{A_{\infty, n}\right\}$ is made using the formula $A_{\infty, n}=\left(A_{n+1}-A_{n}\right) /(\delta-1)$. From the above experimental values of bifurcation points and using $\delta=4.669201609102990671853204$ the sequence of values is constructed as follows:
$A_{\infty, 1}=2.7065667836769114$
$A_{\infty, 2}=2.706567099795308$
$A_{\infty, 3}=2.7065672365325124$
$A_{\infty, 4}=2.706567238299073$
$A_{\infty, 5}=2.7065672384866657$
$A_{\infty, 6}=2.7065672384925636$
$A_{\infty, 7}=2.706567238492908$
$A_{\infty, 8}=2.706567238492924$
$A_{\infty, 9}=2.7065672384929154$
$A_{\infty, 10}=2.7065672384929096$
$A_{\infty, 11}=2.7065672384928487$

region starts.

It may be observed that the sequence converges to the point $2.7065672384929 \ldots$ after which chaotic

\section{References}

[1]. Beddington, J.R., Free, C.A., Lawton, J.H., “Dynamic Complexity in Predator-Prey models framed in difference equations”, Nature, 225(1975), $58-60$.

[2]. Falconer, K.J.,"Fractal Geometry: Mathematical Foundations and Applications”, John Wiley publication.

[3]. Feigenbaum, M.J., "Qualitative Universility for a class of non-linear transformations", J.Statist.Phys,19:1(1978),25-52.

[4]. Feigenbaum, M.J., "Universility Behavior in non-linear systems”, Los Alamos Science,1.(1980),4-27.

[5]. Henon, M., “A two dimensional mapping with a strange attractor”, Comm. Math. Phys. Lett.A 300(2002), 182-188

[6]. Hilborn, R.C., "Chaos and Non-linear dynamics”, Oxford Univ.Press.1994.

[7]. Hone, A.N.W., Irle, M.V.,Thurura, G.W., "On the Neimark-Sacker bifurcation in a discrete predetor-prey system”,2009.

[8]. Kujnetsov, Y., "Elements of Applied Bifurcation Theory", Springer(1998).

[9]. May, R.M., "Simple Mathematical Models With Very Complicated Dynamics", Nature,Vol.261(1976),459.

[10]. Murray, J.D., "Mathematical Biology 1:An Introduction, Third Edition", Springer.

[11]. Murray, J.D., "Mathematical Biology II: Spatial Models and Biomedical Applications”, Springer. 